Artigo Original

\title{
O efeito da prematuridade em habilidades locomotoras e de controle de objetos de crianças de primeira infância ${ }^{1}$
}

\author{
Carolina Maria Coelho Campos \\ Marianne Maila Almeida Soares \\ Maria Teresa Cattuzzo
Programa Associado de Pós-graduação em Educação Física, UPE/UFPB, Recife, PE, Brasil. Escola Superior de Educação Física, Universidade de Pernambuco, Recife, PE, Brasil.

\begin{abstract}
Resumo: Prematuridade é um fator de risco biológico para o desenvolvimento motor infantil, mas não tem sido investigada a interação deste risco com o sexo e a especificidade da tarefa. Objetivou-se verificar o efeito da prematuridade no desempenho locomotor e de controle de objetos em crianças de primeira infância. Vinte meninos e quarenta meninas pré-escolares com idade média de 4,5 anos $(D P=0,7)$ formaram os grupos: Prematuro $(n=30$; média da idade gestacional $=31,7$ semanas, $D P=2,8)$ e Controle ( $n=30$; idade gestacional $>37$ semanas); as habilidades motoras foram avaliadas pelo TGMD-2. De maneira geral, as crianças prematuras foram capazes de atingir o mesmo nível de desempenho motor grosso quando comparadas com aquelas nascidas a termo. Quando as análises levaram em consideração sexo e especificidade da tarefa, os achados sugeriram que ser uma menina prematura afeta negativamente o desenvolvimento em habilidades motoras de controle de objetos.
\end{abstract}

Palavras-chave: Prematuro. Habilidade motora. Pré-escolares.

The effect of prematurity on locomotors and object control skills of toddlers

\begin{abstract}
Prematurity is a biological risk factor for infant motor development and the interaction among this risk with sex and task specificity hasn't been investigated yet. The aim was to verify the effect of prematurity in gross motor performance of children in locomotors and object control skills. Twenty boys and forty girls from preschools, with mean age of 4.5 years $(S D=0.7)$, were separated into premature group $(n=30$, mean gestational age $=31.7$ weeks $S D=2.8)$ and control group $(n=30$, gestational age $>37$ weeks $)$ and their gross motor development was assessed by TGMD-2. In general, premature children were able to achieve the same level of gross motor performance when compared with children born at term. When the analysis took into account sex and task specificity, the findings suggested that being a girl can to affect the early development of the object control motor skills.
\end{abstract}

Keywords: Premature. Motor skills. Preschoolers.

\section{Introdução}

$\mathrm{Na}$ tentativa de melhorar a qualidade de vida dos indivíduos, pesquisas são realizadas para identificar causas de doenças e dos riscos relativos à saúde. Segundo a Organização Mundial de Saúde define-se como fator de risco qualquer circunstância ligada a um aumento na probabilidade de se desenvolver um processo mórbido (WHO, 2009). O nascimento pré-termo, ou prematuro, caracterizado por um período gestacional menor que 37 semanas (BECK et al., 2010), corresponde a um fator de risco biológico, sendo considerado um dos responsáveis pelas causas perinatais de mortalidade infantil no Brasil

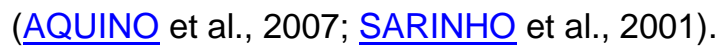

$O$ desenvolvimento motor infantil pode ser considerado um processo multicausal que leva a

\footnotetext{
1 Resultados parciais deste artigo foram apresentados em forma de pôster na Conferência Anual da North American Society for the Psychology of Sport and Physical Activity - NASPSPA - Honolulu, Havaii, Jun, 2012.
}

um aumento na complexidade nas ações motoras, sendo que fatores próprios do organismo, do ambiente e da tarefa, interagem constantemente durante 0 curso do desenvolvimento (SMITH; THELEN, 2003; CATTUZZO et al., 2012). Um fator próprio do organismo que condiciona o desenvolvimento motor é a maturação, um mecanismo inato relacionado à idade cronológica que leva à melhoria de todas as funções orgânicas (GALLAHUE; OZMUN, 2005; HAYWOOD; GETCHELL, 1986). No caso das crianças que nascem com o tempo gestacional inferior a 37 semanas (nascimento pré-termo), mesmo apresentando uma estrutura morfológica completa, eles ainda não possuem a maturação adequada para seu pleno desenvolvimento (PAPALIA; OLDS, 2000; GALLAHUE; OZMUM, 2005).

A prematuridade tem sido considerada um fator de risco biológico para o desenvolvimento 
infantil e, embora os avanços tecnológicos permitam a sobrevida de crianças com idades gestacionais cada vez menores, a imaturidade desses órgãos e sistemas pode ocasionar diversos comprometimentos à saúde da criança (DAVIDSON et al., 2011; GOYEN; LUI, 2009; RESEGUE; PUCCINI; SILVA, 2007). Relativo aos déficits de natureza motora, estudos têm demonstrado que o desenvolvimento motor de prematuros pode apresentar-se com um perfil inferior quando comparado com crianças nascidas a termo (SAGNOL; DEBILLON; DEBÛ, 2007; FORMIGA; CÉSAR; LINHARES, 2010; MAGALHÃES et al., 2009).

De acordo com Malina, Bouchard e Bar-or (2004), os movimentos ativos fetais, que ocorrem principalmente no último trimestre da gestação, são fundamentais para o desenvolvimento motor, sendo considerados como precursores do desenvolvimento locomotor e de controle de objetos na fase pós-natal. Dessa forma, uma vez que as crianças prematuras não passam por essa fase, onde há um maior vigor nos movimentos fetais, é possível esperar que o desenvolvimento motor infantil possa ser comprometido pela prematuridade.

Além disso, segundo Papalia e Olds (2000), fetos do sexo masculino desenvolvem-se mais lentamente, quando comparados aos do sexo feminino, com um atraso de duas semanas em média, já no segundo mês de gestação. Esse atraso pode ser o responsável para que os maiores índices de complicações respiratórias e de risco de mortalidade sejam apresentados pelos meninos prematuros, como os observados nos estudos de Ribeiro et al. (2009), Stevenson et al. (2000) e Cunha et al. (2004). Segundo Malina et al. (2004) os comportamentos motores são bastante específicos para cada idade gestacional e a cada semana a maturação atua melhorando a funcionalidade do organismo. Neste sentido, o atraso típico de desenvolvimento apresentado pelo feto masculino quando combinado com 0 nascimento pré-termo pode aumentar o risco biológico para o desenvolvimento motor de meninos pré-termo.

No entanto, existem evidências de que a sequência progressiva do surgimento de habilidades motoras de prematuros ocorra dentro do limite da normalidade (MANACERO; NUNES, 2008; VOLPI et al., 2010). Concepções desenvolvimentais interacionistas, tais como a paisagem epigenética, sugerem que curso do desenvolvimento dependeria não apenas dos fatores internos dos organismos, mas também das suas relações com os fatores externos. O foco do desenvolvimento seria transferido do gene para as interações que ele realiza com outros agentes, como o ambiente, influenciando e sendo influenciado por ele (NEWELL; LIU; MAYER-KRESS, 2003, PERROTTI; MANOEL, 2001; BARREIROS, 2006). De acordo com a noção de plasticidade fenotípica (GLUCKMAN et al., 2009), essa possibilidade dos indivíduos de ajustarem sua trajetória de desenvolvimento para combinar com seu ambiente, permitiria que aqueles que possuíssem alguma limitação biológica, mas que tivessem um ambiente favorável, conseguissem igualar-se com aqueles sem essa limitação. Assim, pode-se questionar até que ponto 0 desenvolvimento motor seria condicionado pelos fatores intrínsecos ao organismo, no caso, prematuridade e sexo.

Outra questão levantada no presente estudo é se haveria diferenças no desenvolvimento motor grosso de prematuros de acordo com a especificidade da tarefa. Resultados de estudos prévios (MAGALHÃES et al., 2009) tem apontado diferenças em algumas habilidades de controle de objetos entre crianças nascidas pré-termo e a termo, mas não em todas.

Diante do exposto, o objetivo deste estudo foi verificar o efeito da prematuridade em habilidades locomotoras e de controle de objetos, em meninos e meninas de primeira infância.

\section{Métodos}

\section{Caracterização do estudo}

Este é um estudo transversal, não experimental, de comparação entre grupos; foi desenvolvido como um projeto aninhado que utilizou análise secundária de dados epidemiológicos do Estudo Longitudinal de Observação de Saúde e Bem-estar de Crianças em idade Pré-escolar (ELOS-Pré). Os seus procedimentos metodológicos foram aprovados pelo comitê de ética da Universidade de Pernambuco (Registro CEP 097/10; registro CAAE 0096.0.097.000-10) e os participantes tiveram o Termo de Consentimento Livre e Esclarecido assinado pelos pais ou responsável.

\section{Amostra}

Para compor o grupo pré-termo foram selecionadas as crianças de ambos os sexos 
nascidas prematuras e que tivessem completado as duas tentativas de cada uma das doze habilidades motoras grossas analisadas. O grupo controle foi formado por crianças nascidas a termo com idade e sexo semelhantes às crianças do primeiro grupo, pertencentes ao mesmo contexto sociocultural. A equivalência inicial entre os grupos foi garantida por meio da técnica de emparelhamento (SAMPIERI; COLLADO; LUCIO, 2006). As características da amostra são mostradas na Tabela 1.

Tabela1. Caracterização dos participantes com média de idade (anos), massa corporal (kg) e estrutura (cm), e o desvio padrão (DP) dos grupos pré-termo e termo e média da idade gestacional (IG) do grupo pré-termo. Recife, PE

\begin{tabular}{lllccc}
\hline & \multicolumn{2}{c}{ Pré-termo } & \multicolumn{2}{c}{ Termo } & \multirow{2}{*}{ TOTAL } \\
\cline { 2 - 5 } & \multicolumn{1}{c}{$\begin{array}{c}\text { Menino } \\
\mathrm{n}=10\end{array}$} & $\begin{array}{c}\text { Menina } \\
\mathrm{n}=20\end{array}$ & $\begin{array}{c}\text { Menino } \\
\mathrm{n}=10\end{array}$ & $\begin{array}{c}\text { Menina } \\
\mathrm{n}=20\end{array}$ & \\
\cline { 2 - 5 } & & & & \\
Média de idade em anos (DP) & $4,5(0,7)$ & $4,5(0,7)$ & $4,5(0,7)$ & $4,5(0,7)$ & - \\
IG em semanas (DP) & $31,7(2,8)$ & $31,7(2,8)$ & $>37,0$ & $>37,0$ & - \\
Peso em quilogramas (DP) & $18,4(3,6)$ & $19,5(3,8)$ & $20,4(2,5)$ & $20,5(5,9)$ & 0,240 \\
Estatura em centímetros (DP) & $108,5(5,8)^{*}$ & $109(5,8)$ & $113,6(5,0)^{*}$ & $110,3(9,9)$ & 0,210 \\
\hline
\end{tabular}

$\mathrm{DP}=$ desvio padrão; $I \mathrm{G}=$ idade gestacional * Diferença significativa $p \geq 0,05$

\section{Instrumentos e procedimentos}

Para medir o desempenho em habilidades motoras grossas foi utilizada a segunda versão do Test of Gross Motor Development - TGMD-2 (ULRICH, 2000), instrumento composto por seis habilidades locomotoras (correr, galopar, saltitar, saltar o obstáculo, salto horizontal e deslocamento lateral) e seis de controle de objeto (rebater, quicar, receber, chutar, arremessar e rolar a bola). O TGMD-2, que teve sua validade testada para uma população brasileira no estudo de Valentini et al. (2008), apresenta importantes qualidades métricas (fidedignidade, teste-reteste e modelo fatorial confirmatório). No Brasil foi utilizado por diferentes pesquisadores como
Valentini (2002a; 2002b), Valentini et al. (2008), Brauner e Valentini (2009) e Catenassi et al. (2007) indicando ser um instrumento eficiente para avaliar o desenvolvimento motor de crianças entre três e dez anos e onze meses, sendo de fácil compreensão linguística e de baixo custo.

Inicialmente, as tarefas eram explicadas e demonstradas às crianças que, logo depois, realizavam uma tentativa de ensaio e, em seguida, as duas tentativas válidas. Caso houvesse alguma falha no entendimento da tarefa por parte da criança, o instrutor demonstrava mais uma vez e, então, a criança realizava as duas tentativas (Figura 1).

Figura 1. Esquema da aplicação do teste TGMD-2 nas crianças avaliadas. Recife, PE.

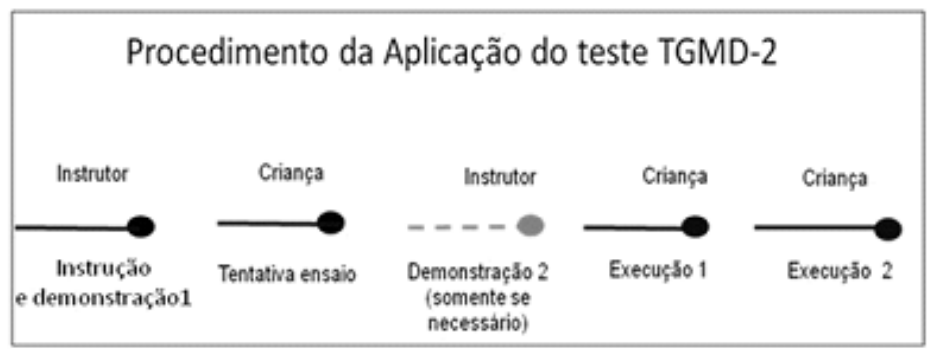

Os desempenhos foram filmados com câmera digital Sony Cyber-Shot DSC-H2O (10.1 Megapixel) e posteriormente analisados em velocidade lenta no Media Player Classic (free download), por dois avaliadores treinados.

$\mathrm{Na}$ decodificação dos dados, as crianças recebiam seus escores de acordo com lista de checagem do teste, atribuindo-se um (1) ponto se a criança atendesse ao critério ou zero (0) ponto se não atendesse. Os escores em cada habilidade eram dados pela soma dos pontos nas duas tentativas. Na decodificação dos dados, a consistência interavaliadores, calculada de acordo com Thomas e Nelson (2002), foi 0,87. As medidas de desempenho usadas foram os escores de cada habilidade, somatório dos escores parciais dos subtestes locomotor (LOC) e 
de controle de objetos (CO) e o escore total, resultante da soma dos subtestes, denominado quociente motor geral (QMG).

\section{Atividades Físicas organizadas}

Para garantir a equivalência inicial entre os grupos, além da técnica de emparelhamento, dados sobre participações em atividades físicas organizadas fora do contexto escolar foram coletados. Essas variáveis, provenientes do banco de dados do projeto ELOS-Pré, indicaram que das 47 crianças prematuras apenas 4 (1 menino e 3 meninas) realizavam atividades físicas organizadas fora do contexto escolar. No grupo Termo, apenas 10 crianças, das 47 analisadas, realizavam atividades físicas organizadas fora do contexto escolar (2 meninos e 8 meninas). Além disso, o teste exato de Fisher garantiu que essa variável não influenciou os resultados, uma vez que não foi constatadas associações entre Atividade Física Organizada e as habilidades motoras grossas para ambos os grupos, $(p>0,05)$.

\section{Peso e estatura}

As variáveis peso e estatura são dados secundários provenientes do banco de dados do projeto ELOS-Pré.

\section{Idade Gestacional}

Os responsáveis pelas crianças incluídas na análise do desempenho de habilidades motoras foram entrevistados por telefone ou pessoalmente sobre a idade de nascimento de seu (sua) filho (a). Essa variável foi computada em semanas (Tabela 1)

\section{Tratamento estatístico}

Após as análises descritivas e verificação da ausência de normalidade dos dados (KolmogorovSmirnov), foi utilizado o teste $\mathrm{U}$ de Mann-Whitney para analisar a diferença entre os escores das habilidades locomotoras e de controle de objetos e entre as médias dos grupos nos escores LOC, CO e QMG, sendo adotado o nível de significância de $p \leq 0,05$. Foram utilizadas planilhas do programa Microsoft Office Excel (2007) e os pacotes estatísticos SPSS 10.0.

\section{Resultados}

Os dados sobre a caracterização dos grupos são apresentados na Tabela 1. Foram realizadas análises comparando as medidas da massa corporal e da estatura das crianças. Os testes não identificaram diferença entre os grupos e sexo com relação à massa das crianças. Já com relação às medidas de estatura foi observada uma diferença marginal $(p=0,057)$ entre os grupos, sendo que meninos prematuros apresentaram uma menor estatura que os meninos nascidos a termo.

Figura 2. Valores da mediana e desvio interquartílico dos escores de desempenho das habilidades de controle de objetos de crianças de 3 a 5 anos. Recife, PE.

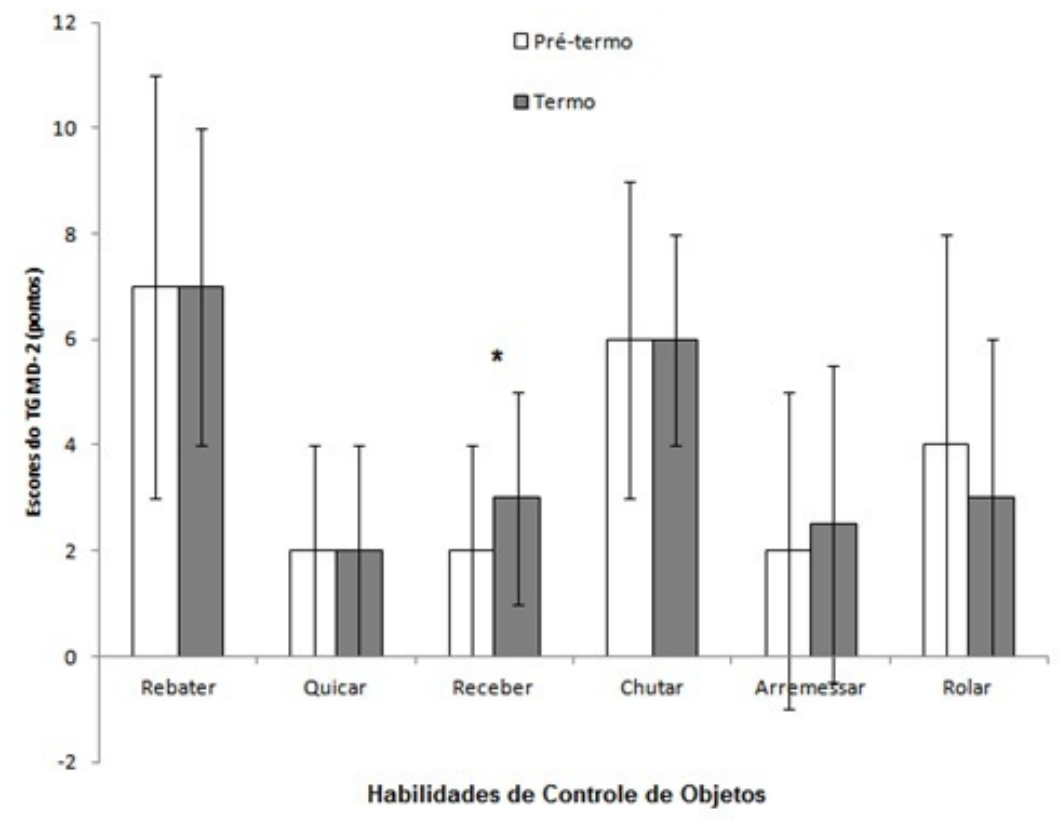

* Diferença Significativa $p \leq 0,05$

Os resultados dos escores dos grupos prétermo e a termo em cada uma das doze habilidades grossas e nos escores parciais de
LOC, CO e o QMG são apresentados nas figuras 2,3 e 4 . 
As análises inferenciais não apontaram diferenças significativas entre os grupos prétermo e a termo nos escores das habilidades locomotoras e nos escores LOC, CO e QMG. Nas habilidades de controle de objetos, o teste $U$ de Mann-Whitney apontou uma diferença significativa $(p=0,014)$ na habilidade de receber a bola sendo as crianças a termo melhores que as crianças pré-termo (Figura 2).

Quando as análises foram estratificadas de acordo com o sexo, entre os meninos dos grupos pré-termo e a termo, não houve diferenças significativas em qualquer das doze habilidades e nos escores LOC, CO e QMG.

Figura 3. Valores da mediana e desvio interquartílico dos escores de desempenho das habilidades de controle de objetos de meninos e meninas de 3 a 5 anos, nascidos pré-termo. Recife, PE.

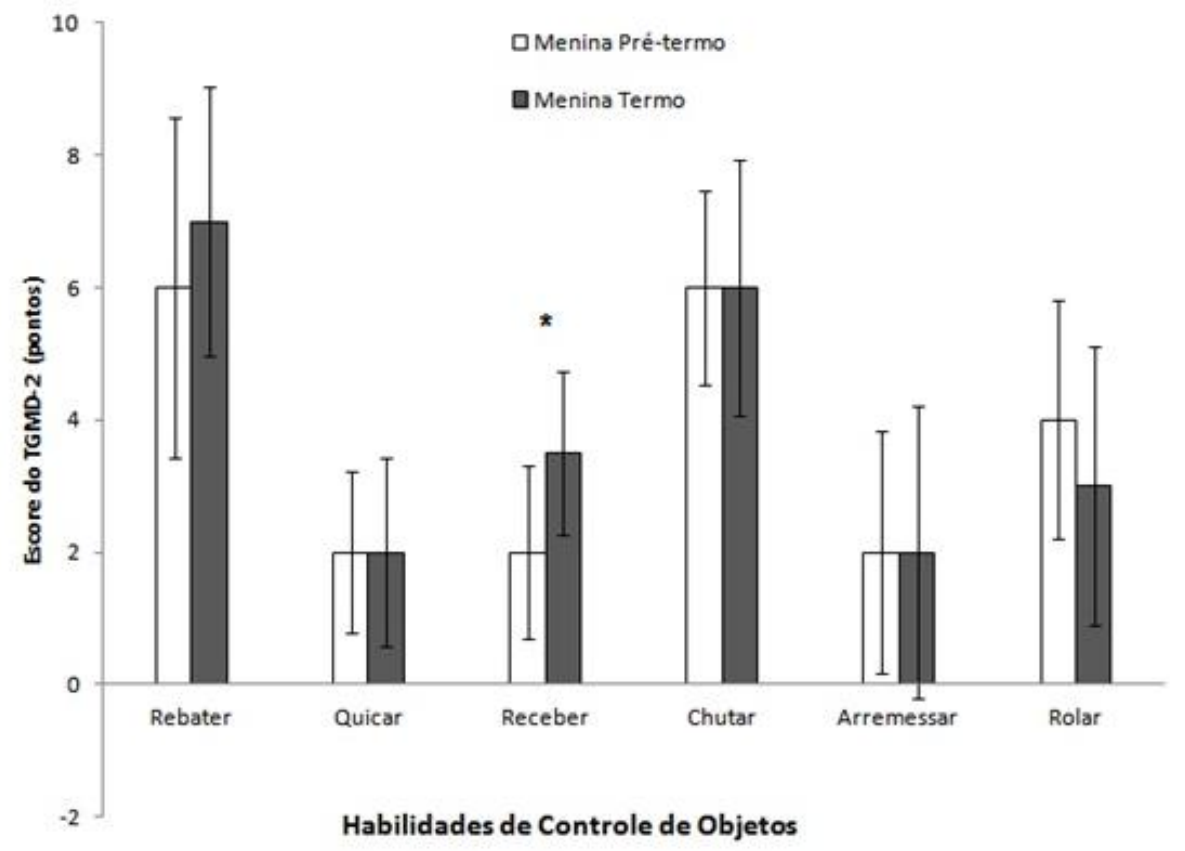

* Diferença Significativa $p \leq 0,05$

Figura 4. Valores da mediana e desvio interquartílico dos escores de desempenho das habilidades locomotoras (LOC), de controle de objetos (CO) e quociente motor geral (QMG) de meninas de 3 a 5 anos nascidos pré-termo e a termo. Recife, PE.

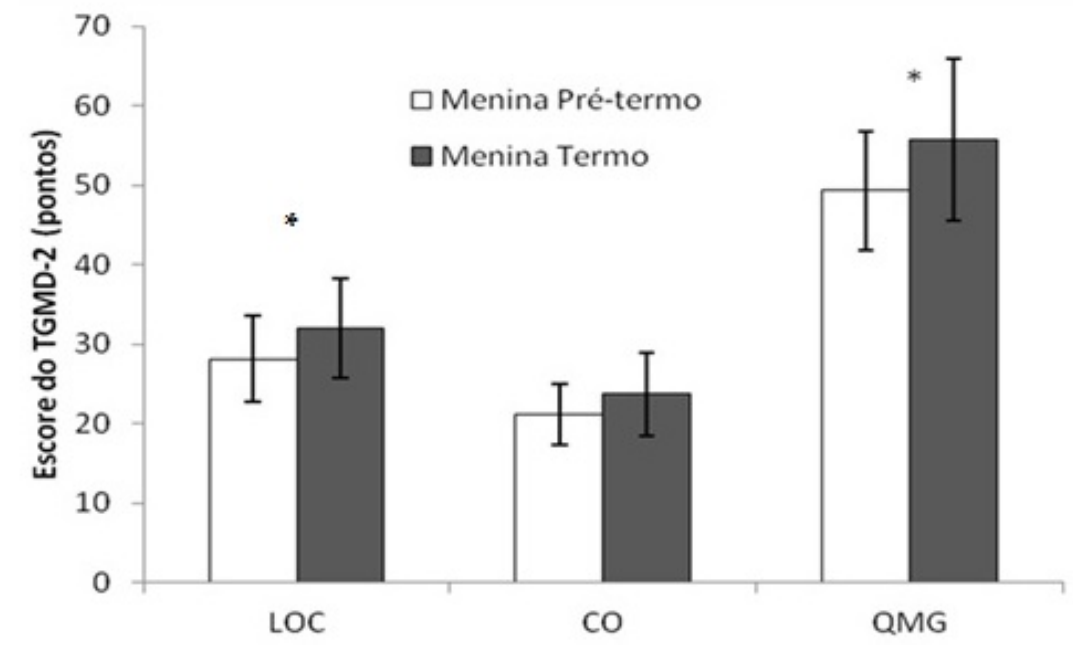

* Diferença Significativa $p \leq 0,05$

Já com relação às meninas, houve diferença significativa na habilidade receber $(p=0,002)$, LOC $(p=0,029)$ e no QMG $(p=0,021)$, com as meninas do grupo termo apresentando um desempenho melhor que as meninas do grupo pré-termo (Figuras 3 e 4).

Por fim, ao serem analisadas somente as crianças do grupo pré-termo, os resultados indicaram que não houve diferença significativa 
entre os sexos nos escores das habilidades locomotoras. Já com relação ao escore de habilidades de controle de objetos, os testes indicaram haver diferença significativa com superioridade dos meninos na habilidade de arremessar $(p=0,038)$ e no escore parcial $C O$ $(p=0,42)$ (Figura 5).

Figura 5. Valores da mediana e desvio interquartílico das habilidades de rebater (Rt), quicar (Q), receber $(\mathrm{RC})$, chutar (Ch), arremessar (A), rolar (Ro) e dos escores parciais de habilidades locomotoras (LOC), de controle de objetos (CO) e quociente motor geral (QMG) de meninos e meninas de 3 a 5 anos nascidos prétermo. Recife, PE.

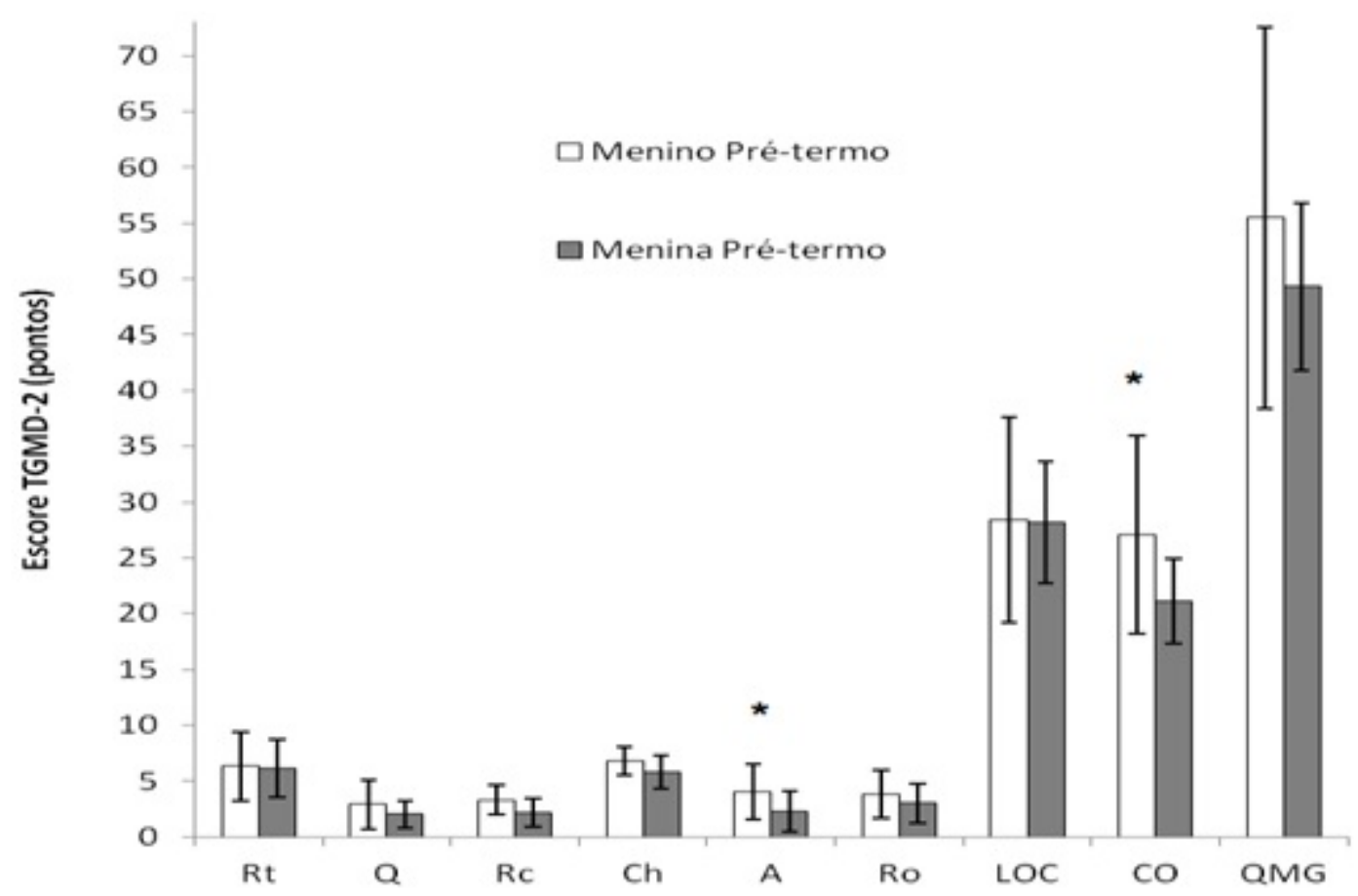

* Diferença significativa ( $p \leq 0,05$ )entre os escores dos grupos.

\section{Discussão}

O objetivo deste estudo foi verificar o efeito da prematuridade em habilidades locomotoras e de controle de objetos, em meninos e meninas de primeira infância. Há que se ressaltar que a correção da idade cronológica em função da prematuridade seria fundamental para 0 diagnóstico do desenvolvimento somente nos primeiros anos de vida. Por exemplo, para um prematuro de 28 semanas não utilizar a idade corrigida, aos dois anos, implicaria em $12 \%$ de diferença em seu desempenho em testes de desenvolvimento, sendo ainda recomendada para prematuros extremos a correção da idade até os três anos de vida (RUGOLO, 2005). Para Volpi et al. (2010) a idade corrigida deve ser usada até obtenção da marcha independente. No caso do presente estudo, as crianças que compõe amostra já ultrapassam esse período crítico, não sendo mais necessária a correção da sua idade cronológica.
Nesta seção, inicialmente, são discutidos os resultados do desempenho motor grosso, comparando os grupos termo e pré-termo. A seguir são discutidas as influências da variável sexo intra e entre grupos e, por fim, a especificidade das tarefas.

Diferenças entre os grupos termo e prétermo

$\mathrm{Na}$ comparação entre os grupos pré-termo e termo, os resultados mostraram que, das doze habilidades analisadas, apenas uma, o receber a bola, foi afetado pela prematuridade. Assim, parcialmente, nossos achados são semelhantes aos resultados de Camargos et al. (2011) no qual as crianças prematuras mostraram desempenhos semelhantes aos seus pares em habilidades motoras grossas.

A falta de diferença entre os grupos na maioria das habilidades investigadas neste estudo permite sugerir que, nas crianças dessa amostra, a 
limitação biológica (prematuridade) não foi suficiente para impedir um desenvolvimento motor grosso satisfatório. Isto talvez porque, mesmo submetido a um insulto biológico, o ser humano seria capaz de explorar outros recursos, sejam do próprio indivíduo ou do ambiente, e construir um comportamento motor mais adequado às suas necessidades (NEWELL; LIU; MAYER-KRESS et al., 2003; PERROTTI; MANOEL, 2001; BARREIROS, 2006).

A paisagem epigenética é uma proposta teórica para o desenvolvimento humano que sugere a influência do ambiente na expressão do código genético; pode ser entendida como o processo individualizado de tomada de decisão do organismo no seu desenvolvimento, uma vez que sua expressão genética seria moldada de acordo com as necessidades do seu ambiente (BARREIROS, 2006; CATTUZZO et al., 2012). De acordo com Newell, Liu e Mayer-Kress et al. (2003) e Barreiros (2006), a visão da paisagem epigenética para o desenvolvimento motor infantil sugere que os mesmos estados de desenvolvimento motor observados em diferentes crianças poderiam ser alcançados por uma variedade de caminhos. Isto então explicaria como as crianças prematuras, mesmo partindo com uma condição desfavorável para o seu desenvolvimento motor, conseguiram atingir escores semelhantes no desenvolvimento motor grosso, quando comparadas as crianças sem a mesma limitação.

Essa explicação também está alinhada à noção de equifinalidade, um dos princípios propostos pela Teoria Geral de Sistemas (BERTALANFFY, 1975). Segundo Bertalanffy (1975), sistemas vivos - como é o caso do ser humano - são sistemas abertos, pois eles mantêm um fluxo constante de entrada e saída de informações e energia, conservando-se mediante a construção e a decomposição de seus componentes; enquanto estão vivos, esses sistemas nunca alcançam o equilíbrio químico e termodinâmico, mantendo-se no estado estacionário. Neste estado, sistemas abertos tem a propriedade de alcançar o mesmo estado final partindo de diferentes condições iniciais e por diferentes maneiras, a chamada equifinalidade (BERTALANFFY, 1975).

\section{Diferença entre os sexos no grupo pré-termo}

Com relação à variação de desempenho entre os sexos no grupo pré-termo, era esperado que ou os meninos tivessem um desempenho motor inferior que as meninas, já que eles seriam os mais afetados pela prematuridade (STEVENSON et al., 2000; CUNHA et al., 2004), ou os resultados acompanhariam os achados de Volpi et al. (2010) onde as aquisições das habilidades motoras não diferiram conforme 0 sexo. Entretanto, nossos resultados apontaram para a superioridade dos meninos pré-termo quando comparados às meninas pré-termo.

A superioridade no desempenho motor de meninos já foi evidenciada nas investigações com crianças com desenvolvimento motor típico (PAIM, 2003; CARVALHAL; VASCONCELOSRAPOSO, 2007). No entanto, também são apresentadas evidências de que as meninas podem ter 0 desempenho motor melhor que meninos, (VAN BEURDEN; BARNETT; DIETRICH, 2002; HARDY et al., 2010).

De acordo Gallahue e Ozmun (2005) e Malina et al. (2004) as diferenças entre meninos e meninas pode estar relacionada aos fatores ambientais. Meninos parecem ser mais encorajados a atividades que envolvam locomoção e em espaços mais amplos, ao contrário das meninas que geralmente vivenciam atividades mais estáticas e verbais do que motoras, como o brincar com a boneca (CARVALHAL; VASCONCELOS-RAPOSO, 2007). A superioridade no desempenho motor não só está relacionada ao sexo do indivíduo, mas também - e talvez principalmente - com tipo de prática de atividades motoras que são estimuladas, de acordo com seu gênero.

De acordo com Hanson e Gluckman (2011) crianças são bastante influenciadas em suas condições iniciais de desenvolvimento, o que nos faz sugerir que a superioridade dos meninos prematuros, quando comparados às meninas prematuras, parece estar mais relacionada ao ambiente em que essas crianças estão envolvidas. Neste sentido, a partir de nossos achados, poder-se-ia especular se as restrições ambientais teriam sido favoráveis para o comportamento motor grosso de meninos, de modo a neutralizar o déficit biológico apresentado pelo nascimento pré-termo.

Diferença entre os sexos e grupos: interação entre prematuridade e sexo

As meninas do grupo pré-termo, mostraram desempenho inferior às meninas do grupo termo, 
concordando com os achados de Gäddlin et al. (2008), Caravale et al. (2005), Vieira e Linhares (2011), Saccani e Valentini (2010), Felice et al. (2010), Van Baar et al. (2005) e Goyen e Lui (2009), nos quais crianças prematuras também tiveram seu desenvolvimento motor influenciado por tal condição.

De acordo com as proposições de Goyen e Lui (2002), Rabinovich e Carvalho (2001), Brauner e Valentini (2009) e Monteiro (2006) sobre a importância do ambiente e fundamentando-se na visão da paisagem epigenética, poder-se-ia especular se, para as meninas, as restrições ambientais teriam sido desfavoráveis para seu desenvolvimento motor grosso, potencializando as limitações trazidas pela prematuridade.

\section{Especificidade das tarefas}

Relativo aos resultados dos escores parciais, as meninas termo mostraram ser superiores às meninas pré-termo no escore parcial de habilidades locomotoras, contrariando os resultados dos estudos de Camargos et al. (2011)

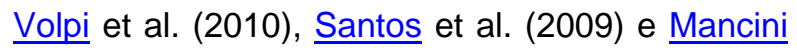
et al. (2002), que ao analisarem habilidades de locomoção não encontraram diferenças significativas entre crianças pré-termo e termo.

A classe de habilidades locomotoras pode ser caracterizada como aquela que envolve 0 deslocamento do corpo no espaço (GALLAHUE; OZMUN, 2005) e exige movimentos dinâmicos e ativos, o que, muitas vezes é reprimido pelos pais, o que pode significar uma limitação para o desenvolvimento de habilidades motoras. Características físicas do ambiente como ausência de espaços abertos, segurança, entre outros, talvez também possam ser pensados como obstáculos para a o engajamento em atividades motoras. Assim, considerando que nossos resultados contrariam estudos anteriores, mais uma vez poder-se-ia especular se, neste estudo, as restrições ambientais teriam sido desfavoráveis para o desenvolvimento locomotor grosso de meninas, potencializando as limitações trazidas pela prematuridade.

A prática organizada de habilidades motoras é um importante fator para que crianças atinjam melhores níveis de desempenho motor. Segundo Clark (2007) o desenvolvimento de habilidades motoras requer prática e instruções específicas. Uma recente meta-análise comprovou o efeito positivo de intervenções com programas de habilidades motoras no comportamento motor de crianças (LOGAN et al., 2011).

Com relação ao desempenho específico por habilidade parece que as tarefas de controle de objetos ofereceram maior dificuldade para as crianças pré-termo. Especificamente nas habilidades de arremessar e receber a bola, o resultado foi semelhante ao estudo de Magalhães et al. (2009) no qual as crianças prematuras também tiveram baixos desempenhos no escore total de atividades com bolas. Magalhães et al. (2009) e Camargos et al. (2011), em seus estudos, ainda apontam a área de destreza manual como a de pior desempenho das crianças pré-termo.

Em termos das capacidades motoras que fundamentam as habilidades (SCHMIDT; LEE, 1999), o arremessar e o receber a bola exigem velocidade nos movimentos de manipulação de objetos com as mãos e braços, bem como precisão nos ajustes em grandes grupos musculares. Além disso, ao executar esses tipos de habilidades, os indivíduos precisam lidar com diferentes tipos de informações ao mesmo tempo. A especificidade da tarefa, tamanho, peso ou tipo de bola utilizada e, especialmente para a habilidade de receber, na qual o ajuste para a direção e trajetória da bola exige antecipação, podem ter proporcionado maior dificuldade às crianças.

Finalmente, algumas sugestões para outros estudos podem ser apontadas. Nas próximas investigações poderia ser utilizada uma análise com o dado contínuo da idade gestacional das crianças envolvidas, buscando conhecer mais sobre a extensão das restrições advindas da prematuridade. Também poderiam ser analisadas habilidades que exigissem o controle motor de pequenos grupos musculares (exigência de precisão) e habilidades que exigissem a antecipação, pois essas especificidades da tarefa poderiam - talvez - expor melhor as diferenças entre prematuros e nascidos a termo. Além disso, a análise da história de vida da criança também poderia ser uma fonte importante de informações: se conhecêssemos a trajetória individual de cada uma delas e seu cotidiano, poderíamos conhecer as influências importantes do ambiente no seu desenvolvimento. 


\section{Conclusões}

O conjunto de resultados do presente estudo permite concluir que o desenvolvimento motor grosso das crianças recifenses de primeira infância foi afetado apenas parcialmente pela prematuridade. No entanto, quando as análises levam em consideração o sexo e a especificidade das tarefas pode-se concluir que a prematuridade interagiu com esses fatores. Nesta amostra de crianças, ser prematura e do sexo feminino afetou negativamente 0 desempenho em habilidades locomotoras e de controle de objetos.

Além disso, nossos resultados nos permitem sugerir que 0 ambiente em que essas crianças estavam envolvidas pode ter sido um fator influenciando o rumo do desenvolvimento de crianças prematuras.

As informações fornecidas por esta pesquisa permitem que profissionais de saúde que convivem com crianças com essas características, em especial aos professores de Educação Física, possam dispor de conhecimentos mais precisos sobre 0 desenvolvimento motor de crianças em situação de risco.

\section{Referências}

AQUINO, T. A.;GUIMARÃES, M.F.J; SARINHO, S.W.; FERREIRA, L.O.C. Fatores de risco para a mortalidade perinatal no Recife, Pernambuco, Brasil, 2003. Cadernos de Saúde Pública, Rio de Janeiro, v. 23, n.12, p. 2853-2861, 2007. Disponível em:

<http://www.scielo.br/pdf/csp/v23n12/05.pdf> Acesso em 11 de maio de 2011.

BARREIROS, J. Um Olhar sobre o desenvolvimento das acções: a paisagem epigenética. In D. Rodrigues (Ed). 0 corpo que (des)conhecemos, p. 99-108. Lisboa: Edições FMH, 2006. Disponível em:

<http://www.fmh.utl.pt/Cmotricidade/dm/textosjb/te xto 2.pdf > Acesso em 11 de março de 2011.

BECK, S.; WOJDYLA, D.; SAY, L.; BETRAN, A.P.; MERIALDI, M.; REQUEJO, J.H.; RUBENS, C.; MENON, R.; VAN LOOK, P.F. A. The worldwide incidence of preterm birth: a systematic review of maternal mortality and morbidity. Bull World Health Organ, v. 88, p. 31-38, 2010. Disponível em:

$<$ http://www.who.int/reproductivehealth/publication s/monitoring/preterm birth/en/> Acesso em 18 de maio de 2011.
BERTALANFFY, L.V.Teoria Geral dos Sistemas, 2a ed. Petrópolis: Vozes, 1975.

BRAUNER, L.M.; VALENTINI, N.C. Análise do desempenho motor de crianças participantes de um programa de atividades físicas. Revista da Educação Física / UEM, v. 20, n. 2, p. 205-216, 2009. Disponível em: <

http://periodicos.uem.br/ojs/index.php/RevEducFis larticle/view/6070/4430> Acesso em 18 de maio de 2011.

CAMARGOS, A.C.R.; FONTES, P.L.B.; ARAÚJO, A.P.S.; SILVA, F.C.; PEREIRA, L.P.; SOUZA S.M.F. Desenvolvimento motor de crianças prétermo moderadas aos sete e oito anos de idade. Fisioterapia e Pesquisa, v. 1, n. 2, p. 182-187, 2011. DOl< http://dx.doi.org/10.1590/S1809$\underline{29502011000200014>}$

CARAVALE, B.; TOZZI, C.; ALBINO, G.; VICARI, $S$. Cognitive development in low risk preterm infants at 3-4 years of life. Archives of Disease in Childhood Fetal and Neonatal, v. 90, 2005. Disponível em:<http://fn.bmj.com/content/90/6/F474.full.pdf+ html> Acesso em: 03 de outubro de 2012.

CARVALHAL, M.; VASCONCELOS-RAPOUSO, $J$. Diferenças entre gêneros nas habilidades: correr, saltar, lançar e chutar. Motricidade, v. 3, n. 3, p. 44-56, 2007. Disponível em: $<$ http://www.redalyc.org/redalyc/pdf/2730/2730204 86006.pdf> Acesso em 11 de março de 2011.

CATENASSI, F.Z.; MARQUES, I.; BASTOS, C.B.; BASSO, L.; RONQUE, E.R.V.; GERAGE, A.M. Relação entre índice de massa corporal e habilidade motora grossa em crianças de quatro a seis anos. Revista Brasileira de Medicina do Esporte, v.13, n. 4, p. 227-230, 2007. Disponível: $<$ http://www.scielo.br/pdf/rbme/v13n4/0 3.pdf> Acesso em: 03 de outubro de 2012.

CATTUZZO, M.T.; BELTRÃO, N.B.; CAMPOS, C.M.C; GUERRA, E. A multicausalidade desenvolvimental: uma abordagem sistêmica da proficiência motora e da prática da atividade física. In M.T. CATTUZZO; I.O. CAMINHA (Org.). Fazer e pensar ciência em Educação Física Livro 1 (cap. 3). Paraíba: Editora da UFPB, 2012.

CLARK, J.E. On the Problem of Motor Skill Development. JOPERD, v. 78, n. 5, p. 39-44, 2007.

CUNHA, A. A.; FERNANDES, D. S.; MELO, P.F.; GUEDES, M.H. Fatores Associados à Asfixia Perinatal. Revista Brasileira de Ginecologia e Obstetrícia, v. 26, n. 10, 2004. Disponível em: < http://www.scielo.br/scielo.php?pid=S0100$\underline{72032004001000007 \& \text { script }=\text { sci arttext }>\text { Acesso }}$ em 20 de abril de 2011. 
DAVIDSON, J.; JOÃO, P.C.; RODRIGUES, R.O; SCAVACINI, A. S. Respostas cardiopulmonares durante o esforço em crianças e adolescentes nascidas prematuras. Revista Paulista de Pediatria, v. 29, n. 3, p. 443-448, 2011. Disponível em: $<$ http://www.scielo.br/pdf/rpp/v29n3/a21v29n3.pdf $>$ Acesso em 23 de novembro de 2011.

FELICE, T.D.; RIBEIRO, J.A.A.B.; ISHIZUKA, R.O.R.; GODOY, G.J. Desenvolvimento apendicular de lactentes pré-termos com 9 meses de idade cronológica. Revista Medicina de Reabilitação, v. 29, n. 1, p. 19-22, 2010. Disponível em: < http://files.bvs.br/upload/S/01035894/2010/v29n1/a005.pdf > Acesso em 11 de março de 2011.

FORMIGA, C.K.M.R.; CEZAR, M.E.N.; LINHARES, M.B.M. Avaliação longitudinal do desenvolvimento motor e da habilidade de sentar em crianças nascidas prematuras. Revista de Fisioterapia e Pesquisa, v. 17, n. 2, p. 102-107, 2010. Disponível em:<

http://www.revistasusp.sibi.usp.br/pdf/fpusp/v17n2 102.pdf> Acesso em 11 de março de 2011.

GALLAHUE, D. L.; OZMUN, J. C. Compreendendo o desenvolvimento motor: bebês, crianças, adolescentes e adultos, $3 \mathrm{a}$ ed. São Paulo: Phorte, 2005.

GÄDDLIN, P.; FINNSTRÖM, O.; WANG, C.; LEIJON, I. A fifteen-year follow-up of neurological conditions in VLBW children without overt disability: Relation to gender, neonatal risk factors, and end stage MRI findings. Early Human

Development, v. 84, n. 5, p. 343-349, 2008.

Disponível em:

<http://www.sciencedirect.com/science/article/pii/S 0378378207001727> Acesso em: 03 de outubro de 2012.

GOYEN, T-A; LUI, K. Longitudinal motor development of "apparently normal" high-risk infants at 18 months, 3 and 5 years. Early Human Development, v. 70, p. 103-115, 2002.

Disponível em:

<http://www.ncbi.nlm.nih.gov/pubmed/12441208> Acesso em 11 de março de 2011.

GOYEN, T-A.; LUI, K. Developmental coordination disorder in "apparently normal" schoolchildren born extremely preterm. Archives of Disease in Childhood, v. 94, p. 298-302, 2009.

GLUCKMAN, P. D., et al. Towards a new developmental synthesis: adaptive developmental plasticity and human disease. LANCET, v. 373, p. 1654-1657, 2009. Disponível em: $<$ http://www.ucl.ac.uk/paediatricepidemiology/pdfs/P623.pdf> Acesso em 11 de março de 2011.
HANSON, M.; GLUCKMAN, P.D. Developmental origins of noncommunicable disease: population and public health implications. American Journal of Clinical Nutrition, v. 94, (supl no 6 ), 1754S1758S, 2011.

HARDY, L. L.; KING, L.; FARRELL, L.; MACNIVEN, R.; HOWLETT, S. Fundamental movement skills among Australian preschool children. Journal of Science and Medicine in Sport, v. 13, n. 5, p. 503-508, 2010. Disponível em: < http://sydney.edu.au/medicine/publichealth/panorg/pdfs/Hardy JSMS in\%20press.pdf $>$ Acesso em 11 de março de 2011.

HAYWOOD, K. M.; GETCHELL, N. Life span motor development. Champaign, IL: Human Kinetics, 1986.

LOGAN, S.W.; ROBINSON, L.E.; WILSON, A.E.; LUCAS, W.A. Lucas Getting the fundamentals of movement: a meta-analysis of the effectiveness of motor skill interventions in children. Child: Care, Health and Development, v. 38, n. 3, p. 305-315, 2011.

MAGALHÃES, L.C.; RESENDE, F.C.A; MAGALHÃES, C.M.; ALBUQUERQUE, P.D.R. Análise comparativa da coordenação motora de crianças nascidas a termo e pré-termo, aos 7 anos de idade. Revista Brasileira de Saúde Materno Infantil v. 9, n. 3, p. 293-300, 2009. Disponível em:

$<$ http://www.scielo.br/scielo.php?pid=S1519$38292009000300008 \&$ script=sci arttext $>$ Acesso em 11 de março de 2011.

MALINA, R. M.; BOUCHARD, C.; BAR-OR, O. Growth, maturation, and physical activity, $2 \mathrm{a}$ ed. Champaign: Human Kinetics Publishers, 2004.

MANACERO, S.; NUNES, M.L. Evaluation of motor performance of preterm newborns during the first months of life using the Alberta Infant Motor Scale (AIMS). Jornal de Pediatria, v. 84, n. 1, 2008. Disponível em: <http://www.ncbi.nlm.nih.gov/pubmed/18202750> Acesso em 18 de maio de 2011.

MANCINI, M.C. et al. Estudo do desenvolvimento da função motora aos 8 e 12 meses de idade em crianças nascidas pré-termo e a termo. Arquivos de Neuropsiquiatria, v. 60 , n. 4, p. 974-980, 2002. Disponível em: $<$ http://www.scielo.br/scielo.php?pid=S0004282X2002000600017\&script=sci arttext $>$ Acesso em 18 de maio de 2011

MONTEIRO, M. Desenvolvimento Motor em contexto: um desafio de pesquisa para profissionais de Educação Física. Revista Brasileira de Educação física e Esporte, v. 20, (supl. no 5), p. 121-123, 2006. Suplemento n.5. 
Disponível em:

$<$ http://citrus.uspnet.usp.br/eef/uploads/arquivo/33 Anais p121.pdf $>$ Acesso em 18 de maio de 2011

NEWELL, K.M.; LIU, Y.T.; MAYER-KRESS, G. A dynamical systems interpretation of epigenetic landscapes for infant motor development. Infant Behavior and Development, v. 26, p. 449-472, 2003. Disponível em:

$<$ http://fulguratio.files.wordpress.com/2011/09/new ell-2003-a-dynamical-systems.pdf> Acesso em 03 de setembro de 2011

PAIM, M.C.C. Desenvolvimento motor de crianças pré-escolares 39 entre 5 e 6 anos. Lecturas, Educación Física y Deportes, v. 8, n. 58, p. 2003. Disponível em:

<http://www.efdeportes.com/efd58/5anos.htm>

Acesso em 18 de maio de 2011.

PAPALIA, D. E.; OLDS, S.W. Desenvolvimento Humano, 7a ed., Porto Alegre: Artes Médicas, 2000.

PERROTTI, A.C.; MANOEL, E.J. Uma visão epigenética do desenvolvimento motor. Revista Brasileira de Ciência e Movimento, v. 9, n. 4, 2001. Disponível em:

$<$ http://www.unifra.br/professores/anabonini/Comp ortamento\%20Motor.pdf> Acesso em 18 de maio de 2011.

RABINOVICH, E.P.; CARVALHO, A.M.A. Modo de morar e modo de cuidar: uma proposta de tipologia. Revista Psicologia Ciência e

Profissão, v. 21, n. 2, p. 74-85, 2001. Disponível em:<http://pepsic.bvsalud.org/scielo.php?pid=S14 14-98932001000200009\&script=sci arttext> Acesso em 18 de maio de 2011.

RESEGUE, R.; PUCCINI, R.F.; SILVA, E.M.K. Fatores de risco associados a alterações no desenvolvimento da criança. Pediatria, v. 29, n. 2 , 117-128, 2007.

RIBEIRO, A.M.; GUIMARÃES, M.J.; LIMA, M.C.; SARINHO, S.W.; COUTINHO, S.B. Fatores de risco para mortalidade neonatal em crianças com baixo peso ao nascer. Revista de Saúde

Pública, v. 43, n. 2, p. 246-255, 2009. Disponível em: <http://www.scielo.br/pdf/rsp/v43n2/6833.pdf> Acesso em 18 de maio de 2011

RUGOLO, L.M.S.S. Crescimento e desenvolvimento a longo prazo do prematuro extremo. Jornal de Pediatria, v. 81, (Supl. oㅜ 1), S101-S110, 2005. Disponível em: $<$ http://www.scielo.br/pdf/jped/v81n1s1/v81n1s1a1 3.pdf> Acesso em: 03 de outubro de 2012.

SACCANI, R.; VALENTINI, N.C. Análise do desenvolvimento motor de crianças de zero a 18 meses de idade: representatividade dos itens da Alberta Infant Motor Scale por faixa etária e postura. Revista Brasileira de Crescimento e Desenvolvimento Humano, v. 20, n. 3, p. 711722, 2010. Disponível em:

$<$ http://www.revistasusp.sibi.usp.br/pdf/rbcdh/v20n 3/06.pdf> Acesso em 18 de maio de 2011

SAGNOL, C.; DEBILLON, T.; DEBÛ, B. Assessment of motor control using kinematics analysis in preschool children born very preterm. Developmental Psychobiology, v. 49, n. 4, p. 421-432, 2007.

SAMPIERI; R.H.; COLLADO, C.F; LUCIO, P.B. Metodologia de pesquisa, 3a ed. São Paulo: McGraw-Hill, 2006.

SANTOS, D.C.C.; TOLOCKA, R.E.; CARVALHO, J.; HERINGER, L.R.C.; ALMEIDA, C.M.; MIQUELOTE, A.F. Desempenho motor grosso e sua associação com fatores neonatais, familiares e de exposição à creche em crianças até três anos de idade. Revista Brasileira de

Fisioterapia, v. 13, n. 2, p. 173-179, 2009.

Disponível em:

$<$ http://www.scielo.br/pdf/rbfis/v13n2/aop023 09.p df> Acesso em 18 de maio de 2011

SARINHO, S. W.; MELO, D.A.F.; SILVA, G.A.P.; LIMA, M.C. Fatores de risco para óbitos neonatais no Recife: um estudo caso-controle. Jornal de Pediatria, v. 77, n. 4, 2001. Disponível em: < http://www.scielo.br/pdf/jped/v77n4/v77n4a11.pdf> Acesso em 17 de maio de 2011.

SCHMIDT, R. A; LEE,T.D. Motor control and learning: a behavioral emphasis, $3 \mathrm{a}$ ed., Champaign, IL: Human Kinetics Publishers, 1999.

SMITH, L.B; THELEN, E. Development as a dynamic system. Trends in Cognitive Sciences, v. 7, n. 8, 2003. Disponível em: < http://defiant.ssc.uwo.ca/graduate/psych9440/read ings/smith\&theolen.pdf> Acesso em 18 de maio de 2011

STEVENSON, D.K. et al. Sex differences in outcomes of very low birthweight infants: the newborn male disadvantage. Archives of Disease in Childhood-fetal and Neonatal, v.83, p. 182-185, 2000. Disponível em: < http://www.ncbi.nlm.nih.gov/pmc/articles/PMC172 1180/pdf/v083p0F182.pdf> Acesso em 18 de maio de 2011

THOMAS, J.; NELSON, J. Métodos de pesquisa em atividade física e saúde, 3a ed. São Paulo: Artmed Editora, 2002.

ULRICH, D. A. Test of gross motor development - 2. Austin: Prod-Ed, 2000. 
VALENTINI, N.C. A influência de uma intervenção motora no desempenho motor e na percepção de competência de crianças com atrasos motores.

Revista Paulista de Educação Física, v. 16, n. 1, p. 61-75, 2002a. Disponível em: < http://citrus.uspnet.usp.br/eef/uploads/arquivo/v16 \%20n1\%20artigo7(1).pdf>. Acesso em 18 de maio de 2011.

VALENTINI, N.C. Percepções de competência e desenvolvimento motor de meninos e meninas: um estudo transversal. Revista Movimento (ESEF/UFRGS), v. 8, n. 2, p. 51-62, 2002b.

VALENTINI, N.C.; BARBOSA, M.L.L.; CINI, G.V.; PICK, R.K.; SPESSATO, B.C.; BALBINOTTI, M.A.A. Teste de desenvolvimento motor grosso: validade e consistência interna para uma população gaúcha. Revista Brasileira de Cineantropometria e Desempenho Humano, v. 10, 4, p. 399-404, 2008.

VAN BAAR, A.L.; VAN WASSENAER, A.G.; BRIËT, J.M.; DEKKER, F.W.; KOK, J.H. Very Preterm Birth is Associated with Disabilities in Multiple Developmental Domains. Journal of Pediatric Psychology, v. 30, n. 3, p. 247-255, 2005. Disponível em:

$<$ http://jpepsy.oxfordjournals.org/content/30/3/247. full.pdf+html>.

VAN BEURDEN, E.; ZASK, A.; BARNETT, L.M.; DIETRICH,U.C. Fundamental movement skillsHow do primary school children perform? The Move it Groove it'program in rural Australia. Journal of Science and Medicine in Sport, v. 5, n. 3, p. 244-252, 2002.

VIEIRA, M. E. B.; LINHARES, M.B.M. Desenvolvimento e qualidade de vida em crianças nascidas pré-termo em idades pré-escolar e escolar. Jornal de Pediatria, v. 87, n. 4, 2011. Disponível em:

$<$ http://www.scielo.br/scielo.php?pid=S0021$7 \overline{5572011000400003 \& \text { script }=\text { sci arttext }>\text {. Acesso }}$ em: 03 de outubro de 2012.

VOLPI, S.C.P.; RUGOLO, L.M.S.S.; PERAÇOLI, J.C.; CORRENTE, J.E. Acquisition of motor abilities up to independent walking in very low birth weight preterm infants. Jornal de Pediatria, v. 86, n. 2, p. 143-148, 2010. Disponível em: < http://www.scielo.br/pdf/jped/v86n2/en v86n2a11. pdf $>>$ Acesso em 25 de junho de 2011.

WHO. Global Health Risk: Mortality and burden of disease attributable to selected major risks. 2009. Disponível em:< http://www.who.int/healthinfo/global burden disea se/GlobalHealthRisks report full.pdf>. Acesso em 25 de junho de 2011.
Este trabalho teve o apoio da FACEPE, mediante bolsa de iniciação científica da aluna Marianne M.A. Soares (BIC-0265-4.09/11).

\section{Endereço:}

Maria Teresa Cattuzzo

Escola Superior de Educação Física, Campus Universitário HUOC.

Rua Arnóbio Marques, 310 Sto. Amaro

Recife PE Brasil

50100-130

Fax: (81) 3183-3354

e-mail: mtcattuzzo@hotmail.com

Recebido em: 3 de maio de 2012.

Aceito em: 10 de dezembro de 2012.

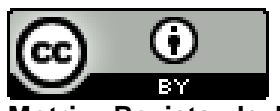

Motriz. Revista de Educação Física. UNESP, Rio Claro, SP, Brasil - elSSN: 1980-6574 - está licenciada sob Creative Commons - Atribuição 3.0 